\title{
Qualidade de vida e saúde bucal em crianças e adolescentes: aspectos conceituais e metodológicos
}

\section{| ${ }^{1}$ Taís de Souza Barbosa, ${ }^{2}$ Fábio Luiz Mialhe, ${ }^{3}$ Aline Rogéria Freire}

de Castilho, ${ }^{4}$ Maria Beatriz Duarte Gavião|

Abstract: O interesse pela qualidade de vida e saúde bucal em crianças e adolescentes vem aumentando, já que as desordens orais provavelmente apresentam efeito negativo na qualidade de vida das mesmas. Neste sentido, questionários que visam a avaliar o impacto da saúde bucal no bem-estar têm sido desenvolvidos e adaptados para este grupo específico. O objetivo deste trabalho é descrever a relação entre qualidade de vida e saúde bucal em crianças e adolescentes, focalizando aspectos conceituais e metodológicos. Os instrumentos desenvolvidos para avaliar a qualidade de vida e saúde bucal em crianças e adolescentes são: o Child Perceptions Questionnaire (CPQ), para crianças de 8-10 $\left(\mathrm{CPQ}_{8 \text { - }}\right.$ $\left.{ }_{10}\right)$ e 11-14 anos $\left(\mathrm{CPQ}_{11-14}\right)$; o Child-Oral Impacts on Daily Performances, para a idade de 11-12 anos; o Child Oral Health Impact Profile, para crianças de 8-14 anos, e o Early Childhood Oral Health Impact Scale para a faixa etária de 2-5 anos. Apesar dos progressos nessa área, ainda existem muitas questôes a serem debatidas e respondidas, como a necessidade de questionários específicos para diferentes idades, a participação dos responsáveis como respondentes secundários e a avaliação do impacto da saúde bucal da criança e do adolescente na família.

> Palavras-chave: adolescente; criança; qualidade de vida; saúde bucal.
1 Mestre em Odontopediatria (FOP/UNICAMP); Departamento de Odontologia Infantil, Área de Odontopediatria, Faculdade de Odontologia de Piracicaba. Endereço eletrônico: tais_sb@ yahoo.com

2 Professor Livre Docente em Odontologia Social (FOP-

UNICAMP); Departamento de Odontologia Social, Faculdade de Odontologia de Piracicaba. Endereço eletrônico: mialhe@ fop.unicamp.br

${ }^{3}$ Mestre em Biologia Oral (Universidade do Sagrado Coração); Departamento de Odontologia Infantil, Área de Odontopediatria, Faculdade de Odontologia de Piracicaba. Endereço eletrônico: alinecastilho@yahoo.com.br

${ }^{4}$ Professor Titular em Odontopediatria (FOPUNICAMP); Departamento de Odontologia Infantil, Área de Odontopediatria, Faculdade de Odontologia de Piracicaba. Endereço eletrônico: mbgaviao@fop.unicamp.br

Recebido em: 07/07/2009. Aprovado em: 15/09/2009. 


\section{Introdução}

A saúde bucal vinha historicamente sendo avaliada por meio de critérios exclusivamente clínicos, os quais não permitem a determinação do real impacto dos problemas bucais na vida dos indivíduos (LOCKER et al., 2002). A necessidade de determinar a repercussão integral de alterações presentes na cavidade bucal levou ao desenvolvimento dos instrumentos de avaliação da qualidade de vida relacionada à saúde bucal, que são utilizados com frequência cada vez maior em pesquisas odontológicas (JOKOVIC et al., 2002).

Entretanto, os índices desenvolvidos eram voltados, em sua maioria, para pacientes adultos ou idosos (SLADE; SPENCER, 1994; LEÃO; SHEIHAM, 1996; SLADE, 1997a; 1997b; ATCHISON; DOLAN, 1999). A qualidade de vida relacionada à saúde bucal em crianças permaneceu por mais tempo desconhecida, sendo objeto de estudo de instrumentos desenvolvidos mais recentemente (JOKOVIC et al., 2002; 2004; GHERUNPONG et al., 2004; BRODER et al., 2007; PAHEL et al., 2007).

Apesar das grandes conquistas associadas à saúde bucal nas últimas décadas, muitas pessoas em todo o mundo, especialmente as mais pobres, ainda são afetadas por problemas bucais como a cárie e a doença periodontal (PETERSEN, 2003). Na infância, a cárie dentária é considerada a doença mais comum dentre aquelas que não regridem espontaneamente nem são passíveis de cura por intervenções farmacológicas de curto prazo (VARGAS et al., 1998). No Brasil, a cárie dentária acomete $27 \%$ das crianças entre 18 e 36 meses de idade, sendo que este percentual chega a 59,4\% aos cinco anos de idade. Entre as crianças de 12 anos, 70\% possuem pelo menos um dente permanente cariado e, entre os adolescentes de 15 a 19 anos, 90\% apresentam a doença (BRASIL, 2004). Os efeitos negativos da cárie dentária sobre a vida das crianças incluem: dificuldade de mastigar, diminuição do apetite, perda de peso, dificuldade para dormir, alteração no comportamento (irritabilidade e baixa auto-estima) e diminuição do rendimento escolar (AYHAN et al., 1996; ACS et al., 1999; 2001; LOW et al., 1999; FILSTRUP et al., 2003; FEITOSA et al., 2005).

Outros problemas bucais, como os transtornos associados à erupção dentária (AGOSTINI et al., 2001), os traumatismos dentários (TRAEBERT et al., 2004), as patologias dos tecidos moles da boca (AGOSTINI et al., 2001), as oclusopatias (MARQUES et al., 2005), as fissuras labiopalatais (CERQUEIRA 
et al., 2005) e a fluorose dentária (OLIVEIRA; MILBOURNE, 2001) afetam crianças com frequência variável. Entretanto, existe pouca informação a respeito das suas possíveis consequências funcionais, emocionais e sociais (CORTES et al., 2002; OLIVEIRA; SHEIHAM, 2004; MARQUES et al., 2006).

Cabe destacar que os indicadores clínicos tradicionais (morbidade, CPO-D, índice de placa, entre outros) são capazes de avaliar a patologia, mas são limitados quando se pretende avaliar os efeitos da doença (PINTO, 2000), principalmente no caso do indivíduo em desenvolvimento. Assim, merecido destaque tem sido dado à avaliação da qualidade de vida destes pacientes infantis em virtude de este ser um componente auxiliar na mensuração da saúde do paciente. Dentro desse contexto, objetiva-se, através de uma revisão de literatura, descrever a relação entre qualidade de vida e saúde bucal em crianças e adolescentes, focalizando aspectos conceituais e metodológicos.

\section{Qualidade de vida e saúde bucal: conceituação}

A qualidade de vida pode ser definida como "a percepção do indivíduo de sua posição na vida, no contexto da cultura e do sistema de valores nos quais ele vive, e em relação aos seus objetivos, expectativas, padrões e preocupações" (WHO, 1997). O conceito de qualidade de vida, além de compreender uma representação subjetiva da sensação de bem-estar, é multidimensional e inclui tanto dimensões positivas quanto negativas (MCGRATh et al., 2004b; SEIDL; ZANNON, 2004; ASSUMPÇÃO JR. et al., 2000).

Bastos et al. (1996) destacam que, uma vez que a qualidade de vida decorre dos aspectos sociais, econômicos, políticos e culturais de uma sociedade, a problemática das doenças que afetam os indivíduos já não pode mais ser explicada unicamente pelos fatores biológicos que as caracterizam. Classificar a saúde em boa, má ou razoável é também definir a qualidade de vida, pois ela surge das condições da classe social, das relações no trabalho, da alimentação, da moradia, do saneamento básico, do meio ambiente saudável, do acesso à educação, ao transporte, ao lazer, aos serviços de saúde, enfim, de tudo o que diz respeito à vida. Dessa forma, como as doenças se refletem de várias formas na vida do indivíduo, a conceituação do termo saúde adquire uma complexidade muito grande, tendo em vista os vários aspectos que envolvem a vida em sociedade. 
A Organização Mundial de Saúde (OMS) define saúde como: "um estado de completo bem-estar físico, mental e social, e não só a ausência de doença ou de enfermidades" (NUTBEAM, 1996). Tal definição tem gerado inúmeras críticas, por não possibilitar uma compreensão clara do que seja "completo bem-estar" e por transmitir a noção de que saúde é algo tão grande que passa a ser inatingível. Esse é um conceito biológico, pertencente ao paradigma médico, com seu foco em agentes etiológicos e em resultados clínicos, cujas raízes filosóficas do modelo são ancoradas na ideia de dualidade entre corpo e mente, no qual a mente e o corpo são entidades separadas, e a saúde e a doença são vistas como fenômenos estritamente biológicos (LOCKER, 1997).

No entanto, com a busca de uma perspectiva mais holística de saúde e doença, esta visão está mudando, resultando em aumento nas pesquisas com foco no paciente não como um corpo e sim como uma pessoa, dando-se crescente importância às experiências subjetivas do indivíduo e às suas interpretaçóes de saúde e doença (LOCKER, 1997; MIOTTO; BARCELLOS, 2001). Esta mudança de paradigma refletiu-se também na odontologia, levando a um aumento nas pesquisas preocupadas em relacionar as condiçôes bucais a doenças de outras localidades da boca e a consequência da saúde na qualidade de vida (LOCKER, 1997).

Sob a ótica da promoção de saúde, a relação entre qualidade de vida e saúde bucal tem sido motivo de atenção dos profissionais da odontologia, principalmente pela relevância de problemas bucais e dos impactos físicos e psicossociais que ela acarreta na vida das pessoas. Os problemas bucais podem causar dor, desconforto, limitações e outras condições decorrentes de fatores estéticos que afetam a vida social, a alimentação, o exercício de atividades diárias e o bem-estar do indivíduo (LEÃO et al., 1998), acarretando problemas significativos na qualidade de vida do indivíduo, o que torna essencial entender como o indivíduo percebe a própria condição bucal, pois seu comportamento é condicionado por esta percepção (BARRÊTO et al., 2004).

\section{Qualidade de vida e saúde bucal: aspectos metodológicos}

O desenvolvimento de instrumentos que avaliem o bem-estar e a qualidade de vida tem sido cada vez mais enfatizado, havendo uma proliferação de instrumentos de avaliação de qualidade de vida relacionada à saúde (BUCZYNSKI et al., 2008). $\mathrm{Na}$ década de 90, consolidou-se a ideia que, em função das características de 
subjetividade, multidimensionalidade e bipolaridade (WHOQOL, 1995), os

instrumentos de mensuração da qualidade de vida devem considerar a perspectiva das pessoas e das populações e não se restringir à perspectiva de profissionais de saúde e pesquisadores (SEIDL; ZANNON, 2004).

Mulhern et al. (1989) propuseram as seguintes características como essenciais a um instrumento de avaliação de qualidade de vida: (1) incluir a abordagem da função física, desempenho escolar e ocupacional, ajustamento social e autosatisfação; (2) ter sensibilidade para detectar os problemas funcionais mais comuns da população estudada; (3) ser confiável e válido para o grupo de pacientes em que será utilizado; (4) ser breve, simples, fácil de administrar e computar, e reprodutível; (5) valer-se de informação de cuidadores familiares ao trato com o indivíduo avaliado; (6) ser corrigido para a idade, sob normas populacionais; (7) estar adequado para detectar desempenho acima da média; (8) permitir estimativa confiável do funcionamento pré-mórbido; (9) e permitir ao indivíduo capaz de entender o conceito de qualidade de vida ou seus componentes a oportunidade de fornecer sua auto-avaliação.

Geralmente, os indicadores de qualidade de vida associada à saúde são construídos sob a forma de questionários compostos de itens (perguntas) que procuram medir, por meio de respostas organizadas sob a forma de escalas numéricas, o quanto aspectos da vida das pessoas, nos domínios físico, psicológico, material e social, entre outros, são afetados pelas condições de saúde (SEVENHUYSEN; TRUMBLE-WADDELL, 1997; WALLANDER et al., 2001). Logo, um aspecto importante desse tipo de ferramenta é a sua capacidade de representar sob a forma numérica as diferenças entre pessoas e comunidades no que diz respeito à qualidade de vida associada à saúde (SEVENHUYSEN; TRUMBLE-WADDELL, 1997). Porém, não se pode perder de vista que qualidade de vida é um construto que, como tal, não pode ser completamente operacionalizado e diretamente medido. Assim, é importante ressaltar que as medidas numéricas obtidas com a aplicação dos indicadores de qualidade de vida associada à saúde devem ser sempre consideradas índices imperfeitos de um construto subjacente (WALLANDER et al., 2001).

Atualmente existem duas formas de mensurar qualidade de vida, através de instrumentos genéricos e instrumentos específicos. Como os dois instrumentos fornecem informaçôes diferentes, eles podem ser empregados concomitantemente. 
Os genéricos abordam o perfil de saúde ou não, procuram englobar todos os aspectos importantes relacionados à saúde e refletem o impacto de uma doença sobre o indivíduo. Podem ser usados para estudar indivíduos da população geral ou de grupos específicos, como portadores de doenças crônicas. Assim, permitem comparar a qualidade de vida de indivíduos sadios com doentes ou de portadores da mesma doença, vivendo em diferentes contextos sociais e culturais. Como desvantagem, não são sensíveis na detecção de aspectos particulares e específicos da qualidade de vida de uma determinada doença (FAYERS, 2000). Dentre os instrumentos genéricos mais frequentemente utilizados, podemos citar o WHOQOL-100 - Instrumento de Avaliação da Qualidade de Vida da Organização Mundial da Saúde (FLECK et al., 1999), o SF-36 (Medical Outcomes Study Questionnaire) (WARE JR; SHERBOURNE, 1992; CICONELLI et al., 1999) e o SIP (Sickness Impact Profile) (BERGNER et al., 1981).

Os instrumentos específicos têm como vantagem a capacidade de detectar particularidades da qualidade de vida em determinadas situaçôes. Eles avaliam, de maneira individual e específica, determinados aspectos de qualidade de vida, como as funçôes física, sexual, o sono, a fadiga, etc. Têm como desvantagem a dificuldade de compreensão do fenômeno e dificuldade de validar as características psicométricas do instrumento (reduzido número de itens e amostras insuficientes) (FAYERS, 2000). Dentre os questionários específicos desenvolvidos para crianças, podemos citar: o AUQEI - Autoquestionnaire Qualité de Vie Enfant Imagé (MANIFICAT et al., 1997; ASSUMPÇÃO JR. et al., 2000) e o CHQ - Child Health Questionnaire (LANDGRAF et al., 1998; MACHADO et al., 2001; RUPERTO et al., 2001).

Os questionários que se destinam a avaliar o impacto dos problemas bucais sobre a qualidade de vida são instrumentos específicos denominados genericamente de indicadores sócio-dentais (COHEN; JAGO, 1976; REISINE, 1981). Suas aplicações são amplas e incluem ações políticas, de pesquisa, de saúde pública e clínicas (WEINTRAUB, 1998; LOCKER, 1996; HAYES, 1998).

Nas últimas duas décadas, vários indicadores sócio-dentais foram desenvolvidos, como o GOHAI - Geriatric Oral Health Assessment Index (ATCHISON; DOLAN, 1999), o DIDL - Dental Impacts on Daily Living (LEÃO; SHEIHAM, 1996), o OHIP - The Oral Health Impact Profile (SLADE; SPENCER, 1994) e a sua versão abreviada - o OHIP-14 (SLADE, 1997a) e o OIDP - Oral Impacts on Daily Performances (SLADE, 1997b). Esses questionários foram construídos para serem aplicados na população adulta, 
porém, recentemente, tanto o OHIP quanto o OIDP foram utilizados em estudos envolvendo adolescentes (BRODER et al., 2000; CORTES et al., 2002; OLIVEIRA; SHEIHAM, 2003; FERREIRA et al., 2004).

Apesar de indicadores sócio-dentais elaborados para adultos terem sido aplicados com sucesso em adolescentes, a percepção de adultos e crianças a respeito do impacto dos problemas de saúde sobre a qualidade de vida é diferente, já que as crianças e os adolescentes possuem uma visão peculiar de si mesmos e do mundo, devido à fase de desenvolvimento físico e emocional em que se encontram (PAL, 1996; KUCZYNSKI; ASSUMPÇÃO JR., 1999; ASSUMPÇÃO JR. et al., 2000). Portanto, o desenvolvimento de instrumentos específicos para crianças viabiliza a mensuração mais acurada do impacto dos problemas bucais sobre a sua qualidade de vida. Levando isso em consideração, Jokovic et al. (2002; 2004) construíram o CPQ (Child Perceptions Questionnaires - $\mathrm{CPQ}_{8-10}$ e $\mathrm{CPQ}_{11}$. ${ }_{14}$ ), Broder et al. (2007) desenvolveram o COHIP (Child Oral Health Impact Profile), Gherunpong et al. (2004) adaptaram o OIDP para uso em crianças criando o Child-OIDP, e Pahel et al. (2007) desenvolveram o ECOHIS - The Early Childhood Oral Health Impact Scale (tabela 1).

O contexto cultural e linguístico no qual o instrumento de avaliação da qualidade de vida é utilizado pode influenciar a validade e confiabilidade dos relatos obtidos. Com poucas exceçôes, os indicadores de qualidade de vida relacionada à saúde são desenvolvidos em língua inglesa e têm como população-alvo indivíduos de países que falam inglês. Porém, a necessidade de utilização desses indicadores em pesquisas é comum a outros países de língua não-inglesa, que têm como opções: criar um novo instrumento ou adaptar o existente em outra língua (GUILLEMIN et al., 1993). Torna-se de importância, portanto, que o instrumento seja traduzido para o idioma de origem do país a ser utilizado e que seja precisamente adaptado às características socioculturais da população a ser analisada, permitindo a avaliação fidedigna. Além disso, é preciso que o instrumento seja facilmente administrado e que não demande tempo na aplicação.

Estudos preliminares confirmaram a validade e confiabilidade do $C \mathrm{CQ}_{1-14}$ em outros países como Inglaterra (MARSHMAN et al., 2005; O’BRIEN et al., 2006), Nova Zelândia (FOSTER-PAGE et al., 2005); Arábia Saudita (BROWN; AL-KHAYAL, 2006), China (MCGRATH et al., 2008; LI et al., 2008), Austrália (DO; SPENCER, 2008) e Brasil (GOURSAND et al., 2008), enquanto as propriedades psicométricas do $\mathrm{CPQ}_{8-10}$ foram confirmadas na Irlanda do Norte 

SPENCER, 2008). O Child-OIDP também já foi adaptado para utilização em outros idiomas: francês (TUBERT-JEANNIN et al., 2005), inglês (YUSUF et al., 2006) e português (CASTRO et al., 2008). Li et al. (2008) e Tesch et al. (2008) adaptaram o ECOHIS desenvolvido nos Estados Unidos para outra cultura, a da França e do Brasil, respectivamente. As propriedades psicométricas do COHIP foram avaliadas apenas nos Estados Unidos (BRODER et al., 2007), onde foi desenvolvido e na Holanda (GEELS et al., 2008). No entanto, no Brasil, ainda não há uma proposta de tradução e validação deste questionário. Tesh et al. (2008) enfatizam que o processo de avaliação da equivalência semântica na adaptação transcultural de instrumentos de qualidade de vida associada à saúde bucal deve ter uma base conceitual e se apoiar em metodologias criteriosamente definidas (tabela 1).

Diversos métodos têm sido propostos para a adaptação transcultural de questionários, variando desde a tradução direta do instrumento à metodologia de tradução/retradução, a qual possui uma combinação de características favoráveis como: capacidade informativa, definida como o grau de indicação objetiva de equivalência semântica pela técnica; transparência da linguagem original, ou o grau em que a técnica fornece informaçôes para o investigador ou para o autor da versão original do instrumento que não tem fluência na linguagem original ou na linguagem alvo; e segurança, ou a oportunidade da técnica em checar o trabalho do tradutor original (BEHLING; LAW, 2000).

\section{Avaliação da qualidade de vida e saúde bucal em crianças e adolescentes}

No caso do indivíduo em desenvolvimento, as propostas para definição de qualidade de vida apresentadas ainda são muito conflitantes e algumas características do universo infantil contribuem para isto. A criança e o adolescente têm diferentes graus de percepção de si mesmos e do mundo, em função da sua fase de desenvolvimento e, com isso, dificilmente podem ser uniformizados numa só concepção de satisfação pessoal (KUCZYNSKI; ASSUMPÇÃO, 1999).

Para crianças e adolescentes, qualidade de vida pode significar "o quanto seus desejos e esperanças se aproximam do que realmente está acontecendo". Também "reflete sua prospecção, tanto para si, quanto para os outros" e "é muito sujeita a alterações sendo influenciada por eventos cotidianos e problemas crônicos" (HINDS, 1990). E ainda, segundo Bradlyn et al. (1996), qualidade de 
vida pode ser definida como multidimensional, pois inclui a interação social, o funcionamento físico e emocional da criança e do adolescente e, quando indicado, de sua família, devendo ser um parâmetro sensível às alterações que ocorram no evoluir do desenvolvimento do indivíduo.

A percepção de saúde e de doença varia conforme a capacidade cognitiva da criança, a qual se modifica de acordo com a idade, em função dos diferentes estágios de desenvolvimento emocional, social e de linguagem (TESCH et al., 2007). Uma estratégia para abordar as diferentes fases de desenvolvimento da criança é criar múltiplas formas de um mesmo instrumento para crianças, cada uma desenhada para diferentes faixas etárias. Esta maneira é vantajosa por levar em consideração os diferentes estágios de desenvolvimento da criança, incluindo sua concepção de doença, assim como sua causa e impacto. Com esta estratégia, é possível desenhar uma série de questionários garantindo que o formato e o conteúdo sejam apropriados para a idade de todas as crianças (MATZA et al., 2004).

Contudo, alguns grupos de crianças, como as muito jovens, as incapacitadas e as gravemente enfermas, apresentam dificuldade para fornecer informação acurada sobre sua qualidade de vida (WALLANDER et al., 2001), devendo os pesquisadores avaliar a idade apropriada do instrumento, incluindo o vocabulário, instruções, estrutura das frases, conteúdo e opções de resposta, preocupando-se com as limitaçôes de desenvolvimento (BUCZYNSKI et al., 2008). Para permitir que mesmo crianças jovens possam responder sobre sua própria qualidade de vida, alguns instrumentos utilizam recursos auxiliares às respostas, como figuras com as quais a criança possa se identificar (ASSUMPÇÃO JR. et al., 2000; GHERUNPONG et al., 2004) ou estórias desenvolvidas em programas computacionais (BULLER, 1999). Outra opção seria o desenvolvimento de um instrumento administrado na forma de entrevista, que pode incluir procedimentos padronizados para ensinar a criança a responder a mensuração e instruindo-a a não responder questôes que não entender, e ainda, checando a compreensão dos itens pela criança. Entretanto, um instrumento administrado por entrevista pode ser mais dispendioso de ser usado que o questionário autoadministrado (BUCZYNSKI et al., 2008). Ainda, para crianças muito jovens, como por exemplo, pré-escolares, os responsáveis podem prover informações que não poderiam ser obtidas de outra forma (PAHEL et al., 2007). Contudo, não se pode garantir que os pais (respondentes secundários) consigam representar bem a realidade das crianças e nem que forneçam respostas verdadeiras, livres de pressão relacionada à aceitabilidade social (MCGRATH et 
al., 2004b). Mesmo assim, a perspectiva dos responsáveis é importante por causa da natureza dependente da relação responsável-criança. É este responsável quem avalia o impacto da saúde da criança e decide se esta deve receber o tratamento; ele pode também informar o impacto da doença da criança e do tratamento no funcionamento familiar, o que é uma parte integral da qualidade de vida relacionada à saúde bucal da criança (MATZA et al., 2004).

Crianças não são seres independentes e a família compreende a rede de indivíduos que dá suporte e afeta de forma mais acentuada a qualidade de vida da criança. No sentido inverso, a própria família é afetada pelos problemas de saúde da criança (PAL, 1996). Familiares de crianças portadoras de doenças crônicas muitas vezes têm suas atividades diárias limitadas e experimentam medos e ansiedades devido aos problemas de saúde da criança. Além disso, os problemas de saúde podem produzir impactos financeiros que tendem a aumentar o estresse familiar (JUNIPER et al., 1996; STEIN; JESSOP, 2003). Portanto, instrumentos desenvolvidos para mensurar a qualidade de vida relacionada à saúde bucal de crianças também devem abordar o impacto desses problemas sobre a qualidade de vida da família (LOCKER et al., 2002).

A avaliação da qualidade de vida relacionada à saúde bucal em criança refletese na percepção dos pacientes sobre sua saúde bucal e com isso pode melhorar a comunicação entre pacientes, pais e a equipe odontológica (WEINTRAUB, 1998). Isto permite um melhor entendimento das consequências do estado de saúde bucal na vida da criança e da vida da sua família (HOLT, 2001), podendo ainda ajudar na priorização de cuidados e estimar a consequência das estratégias de tratamento e iniciativas (SHEIHAM et al., 1982).

\section{Conclusões}

A qualidade de vida relacionada à saúde é um importante componente auxiliar aos indicadores clínicos na avaliação da saúde do paciente. Isto se torna ainda mais relevante em pacientes infantis e com doenças bucais, que determinam um impacto significativo em sua qualidade de vida. Assim, tornam-se cada vez mais necessários instrumentos que auxiliem o cirurgião-dentista e demais profissionais responsáveis por estas crianças e adolescentes para avaliar não só a presença da doença bucal, como também a qualidade de vida desses indivíduos. 


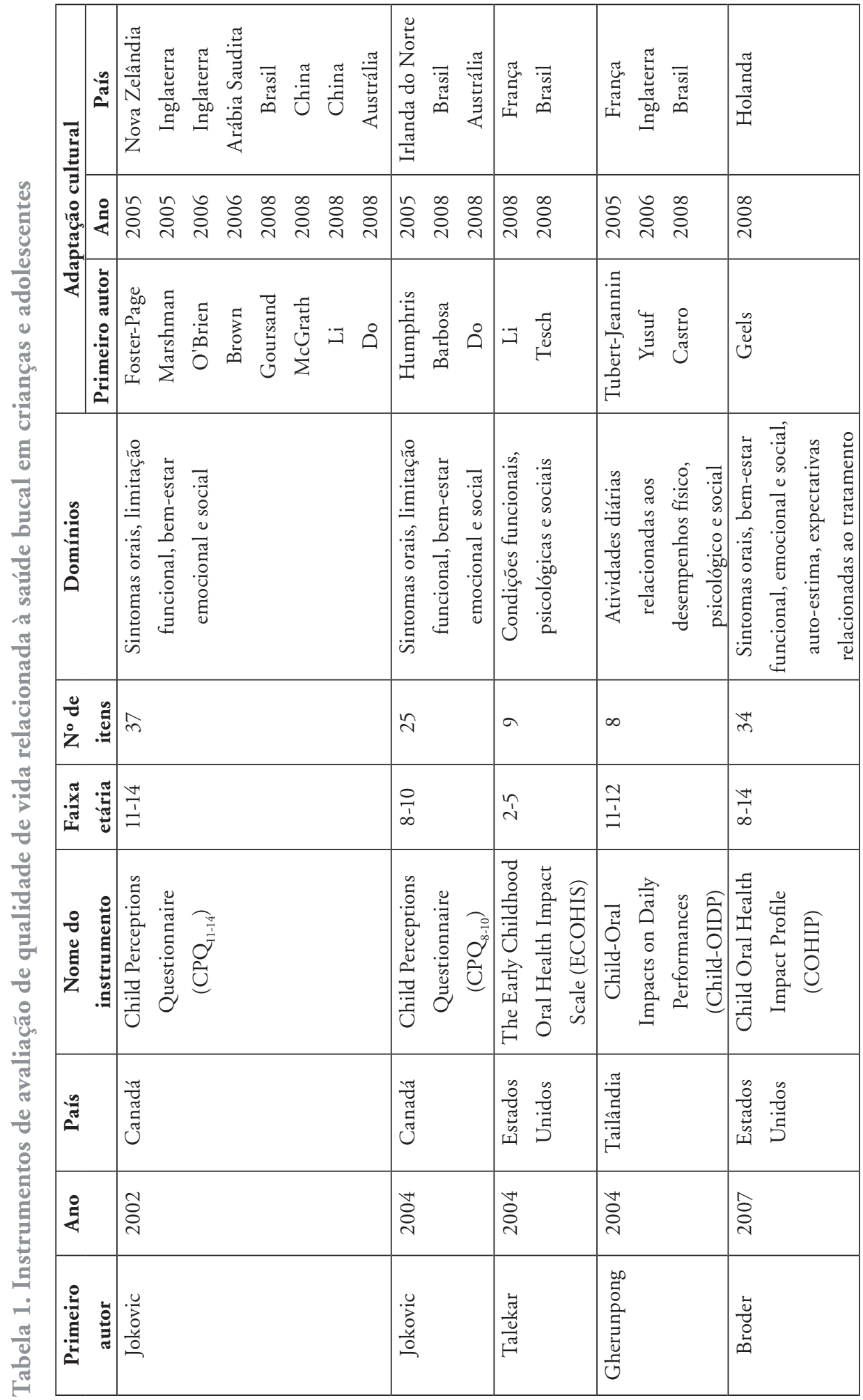




\section{Referências}

ACS, G. et al. Perceived outcomes and parental satisfaction following dental rehabilitation under general anesthesia. Pediatr dent, v. 23, n. 5, p. 419-423, 2001.

ACS, G. et al. The effect of dental rehabilitation on the body weight of children with early childhood caries. Pediatr dent, v. 21, n. 2, p. 109-113, 1999.

AGOSTINI, F.G.; FLAITZ, C.M.; HICKS, M.J. Dental emergencies in a university-based pediatric dentistry postgraduate outpatient clinic: a retrospective study. ASDC J dent child, v. 68, n. 5, p. 316-321, 2001.

ASSUMPÇÃO, J.R. et al. Escala de avaliação de qualidade de vida: AUQEI (Autoquestionnaire Qualité de Vie Enfant Imagé) validade e confiabilidade de uma escala para qualidade de vida em crianças de 4 a 12 anos. Arq neuropsiquiatr, v. 58, n. 1, p. 119-127, 2000.

ATCHISON, K.A.; DOLAN, T.A. Development of the Geriatric Oral Health Assessment Index. J dent educ, v. 54, n. 11, p. 680-687, 1990.

AYHAN, H.; SUSKAN, E.; YILDIRIM, S. The effect of nursing or rampant caries on height, body weight and head circumference. J clin pediatr dent, v. 20, n. 3, p. 209-212, 1996.

BARBOSA, T.S.; SERRA, M.D.; GAVIÃO, M.B.D. Qualidade de vida e saúde bucal em crianças - Parte I: Versão brasileira do Child Perceptions Questionnaire 8-10. Rev C S Col. Disponível em:<http://www.cienciaesaudecoletiva.com.br>. Acesso em: 12 nov. 2008.

BARRÊTO, A.P.R. et al. Qualidade de vida infantil: influência dos hábitos de higiene bucal e do acesso aos serviços odontológicos. Rev ibero-amer odontop odontol bebê, v.7, n.39, p.453-60, 2004.

BASTOS, J.M.; SALIBA, N.A.; UNFER, B. Considerações a respeito de SB e classes sociais. Rev paul odonto, v. 38, n. 4, p. 38-41, 1996.

BEHLING, O.; LAW, K.S. Translating questionnaires and other research instruments: problems and solutions. Thousand Oaks: Sage, 2000.

BERGNER, M. et al. The Sickness Impact Profile: development and final revision of a health status measure. Med Care, v. 19, n. 8, p. 787-805, 1981.

BRADLYN, A.S. et al. Quality of life research in Pediatric Oncology. Research Methods and Barriers. Cancer, v. 78, n. 6, p. 1333-1339, 1996.

BRASIL. Ministério da Saúde. Coordenação Nacional de Saúde Bucal. Projeto SB Brasil 2003 - Condiçôes de saúde bucal da população brasileira: resultados principais. Brasília: Ministério da Saúde, 2004.

BRODER, H.L. et al. Perceived impact of oral health conditions among minority adolescents. J public health dent, v. 60, n. 3, p. 189-192, 2000.

BRODER, H.L.; MCGRATH, C.; CISNEROS, G.J. Questionnaire development: face validity and item impact testing of the Child Oral Health Impact Profile. Community dent oral epidemiol, v. 35, n. 1, p. 8-19, 2007. 
BROWN, A.; AL-KHAYAL, Z. Validity and reliability of the Arabic translation of the child oral health related quality of life questionnaire (CPQ11-14) in Saudi Arabia. Int J paediatr dent, v. 16, n. 6, p. 405-411, 2006.

BUCZYNSKI, A.K.; CASTRO, G.F.; DE SOUZA, I.P.R. O impacto da saúde bucal na qualidade de vida de crianças infectadas pelo HIV: revisão de literatura. Cien saude colet, v. 13, n .6, p. 1797-1805, 2008.

BULLER, H. Assessment of quality of life in the younger child: the use of an animated computer program. J pediatr gastroenterol nutr, v. 28, n. 4, p. 53-55, 1999.

CASTRO, R.A. et al. Child-OIDP index in Brazil: cross-cultural adaptation and validation. Health qual life outcomes, v. 6, p. 68, 2008.

CERQUEIRA, M.N. et al. Ocorrência de fissuras labiopalatais na cidade de São José dos Campos-SP. Rev bras epidemiol, v. 8, n. 2, p. 161-166, 2005.

CICONELLI, R.M. et al. Tradução para a língua portuguesa e validação do questionário genérico de avaliação de qualidade de vida SF-36 (Brasil SF-36). Rev bras reumatol, v. 39, n. 3, p. 143-150, 1999.

COHEN, L.K.; JAGO, J.D. Toward the formulation of sociodental indicators. Int $J$ health serv, v. 6, n. 4, p. 681-698, 1976.

CORTES, M.I.; MARCENES, W.; SHEIHAM, A. Impact of traumatic injuries to the permanent teeth on the oral health-related quality of life in 12-14-year-old children. Community dent oral epidemiol, v. 30, n. 3, p. 193-198, 2002.

DO, L.G.; SPENCER, A.J. Evaluation of oral health-related quality of life questionnaires in a general child population. Community dent health, v. 25, n. 4, p. 205-210, 2008.

FAYERS, Peter M.; MACHIN, David. Quality of life. Assessment, analysis and interpretation. Chichester: John Wiley, 2000.

FEITOSA, S.; COLARES, V.; PINKHAM, J. The psychosocial effects of severe caries in 4-year-old children in Recife, Pernambuco, Brazil. Cad saúde pública, v. 21, n. 5, p. 1550$1556,2005$.

FERREIRA, C.A.; LOUREIRO, C.A.; ARAÚJO, V.E. Propriedades psicométricas de indicador subjetivo aplicado em crianças. Rev saúde pública, v. 38, n. 3, p. 445-52. 2004.

FILSTRUP, S.L. et al. Early childhood caries and quality of life: child and parent perspectives. Pediatr dent, v. 25, n. 5, p. 431-440, 2003.

FLECK, M.P.A.; LEAL, O.F.; LOUZADA, S. Desenvolvimento da versão em português do instrumento de avaliação de qualidade de vida da OMS (WHOQOL-100). Rev bras psiquiatr, v. 21, n. 1, p. 19-28, 1999.

FOSTER-PAGE, L.A. et al. Validation of the Child Perceptions Questionnaire (CPQ 1114). J dent res, v. 84, n. 7, p. 649-652, 2005. 

analysis of the dimensions of the Child Oral Health Impact Profile (Dutch version). EurJ oral sci, v. 116, n. 2, p. 148-152, 2008.

GHERUNPONG, S.; TSAKOS, G.; SHEIHAM, A. Developing and evaluating an oral health-related quality of life index for children; the CHILD-OIDP. Community dent health, v. 21, p. 161-169, 2004.

GOURSAND, D. et al. Cross-cultural adaptation of the Child Perceptions Questionnaire 11-14 (CPQ11-14) for the Brazilian Portuguese language. Health qual life outcomes, v. 14, n. 6, p. 2, 2008.

GUILLEMIN, F.; BOMBARDIER, C.; BEATON, D. Cross-cultural adaptation of healthrelated quality of life measures: literature review and proposed guidelines. J clin epidemiol, v. 46, n. 12, p. 1417-1432, 1993.

HAYES, C. The use of patient based outcome measures in clinical decision making. Community dent health, v. 15, n. 1, p. 19-21, 1998.

HINDS, P. Quality of life in children and adolescents with cancer. Semin oncol nurs, v. 6, n. 4, p. 285-291, 1990.

HOLT, R.D. Advances in dental public health. Prim dent care, v. 8, n. 3, p. 99-102, 2001.

HUMPHRIS, G. B. et al. Oral health-related quality of life for 8-10-year-old children: an assessment of a new measure. Community dent oral epidemiol, v. 33, n. 5, p. 326-332, 2005.

JOKOVIC, A. et al. Questionnaire for measuring oral health-related quality of life in eightto ten-year-old children. Pediatr dent, v. 26, n. 6, p. 512-8, 2004.

JOKOVIC, A. et al. Validity and reliability of a questionnaire for measuring child oralhealth-related quality of life. J dent res, v. 81, n. 7, p. 459-463, 2002.

JUNIPER, E.F. et al. Measuring quality of life in the parents of children with asthma. Qual Life Res, v. 5, n. 1, p. 27-34, 1996.

KUCZYNSKI, E.; ASSUMPÇĀO JR, F.P. Definições atuais sobre o conceito de qualidade de vida na infância e adolescência. Pediatr mod, v. 35, n. 3, p. 73-78, 1999.

LANDGRAF, J.M. et al. Canadian-French, German and UK versions of the Child Health Questionnaire: methodology and preliminary item scaling results. Qual life res, v. 7, n. 5, p. 433-445, 1998.

LEÃO, A.; SHEIHAM, A. The development of a socio-dental measure of dental impacts on daily living. Community Dent Health, v. 13, n. 1, p. 22-26, 1996.

LEÃO, A.T.T.; CIDADE, M.C.; VARELA, J.R. Impactos da saúde periodontal na vida diária. Rev bras odontol, v. 55, n. 4, p. 238-241, 1998.

LI, S.; VERONNEAU, J.; ALLISON, P.J. Validation of a French language version of the Early Childhood Oral Health Impact Scale (ECOHIS). Health qual life outcomes, v. 6, p. 9, 2008. 
LI, X.J. et al. [Validation of a Chinese version of the child perception questionnaire]. Hua Xi Kou Qiang Yi Xue Za Zhi, v. 26, n. 3, p. 267-70, 2008.

LOCKER, D. Applications of self-reported assessments of oral health outcomes. J dent educ, v. 60 , n. 6, p. 494-500, 1996.

LOCKER, D. et al. Family impact of child oral and oro-facial conditions. Community dent oral epidemiol, v. 30, n. 6, p. 438-448, 2002.

LOCKER, David. Concepts of oral health, disease and the quality of life. In: SLADE, G.D. Measuring oral health and quality of life. Chapel Hill: University of North Carolina, 1997. p. 11-23.

LOW, W.; TAN, S.; SCHWARTZ, S. The effect of severe caries on the quality of life in young children. Pediatr dent. v. 21, n. 6, p. 325-326, 1999.

MACHADO, C.S. et al. The Brazilian version of the Childhood Health Assessment Questionnaire (CHAQ) and the Child Health Questionnaire (CHQ). Clin exp rheumatol, v. 19, n. 4, p. 25-29, 2001.

MANIFICAT, S. et al. Evaluation of the quality of life in pediatrics: how to collect the point of view of children. Arch pediatr, v. 4, n. 12, p. 1238-1246, 1997.

MARQUES, L.S. et al. Malocclusion: esthetic impact and quality of life among Brazilian schoolchildren. Am J Orthod Dentofacial Orthop, v. 129, n. 3, p. 424-427, 2006.

MARQUES, L.S. et al. Prevalência da maloclusão e necessidade de tratamento ortodôntico em escolares de 10 a 14 anos de idade em Belo Horizonte, Minas Gerais, Brasil: enfoque psicossocial. Cad saúde pública, v. 21, n. 4, p. 1099-1106, 2005.

MARSHMAN, Z. et al. An evaluation of the Child Perceptions Questionnaire in the UK. Community dent health, v. 22, n. 3, p. 151-155, 2005.

MATZA, L.S. et al. Assessment of health-related quality of life in children: a review of conceptual, methodological, and regulatory issues. Value health, v. 7, n. 1, p. 79-92, 2004.

MCGRATH, C. et al. Translation and evaluation of a Chinese version of the Child Oral Health-related Quality of Life measure. Int J paediatr dent, v. 18, n. 4, p. 267-274, 2008.

MCGRATH, C.; BRODER, H.; WILSON-GENDERSON, M. Assessing the impact of oral health on the life quality of children: implications for research and practice. Community dent oral epidemiol, v. 32, n. 2, p. 81-85, 2004.

MIOTTO, M.H.M.B.; BARCELLOS, L.A. Uma revisão sobre o indicador subjetivo de SB "Oral Health Impact Profile” OHIP. UFES Rev. odontol. Vitória, v. 3, n. 1, p. 32-33, 2001.

MULHERN, R.K.; HOROWITZ, M.E.; OCHS, J. Assessment of quality of life among Pediatric patients with cancer: Psychological assessment. J consult clinic psychol, v. 1, p. 130136, 1989. 
NUTBEAM, Don. Health promotion glossary. In: PAHO. (Org.). Health promotion: an anthology. Washington: Pan American Health Organization, 1996. p. 343-59.

O'BRIEN, C.; BENSON, P.E.; MARSHMAN, Z. Evaluation of a quality of life measure for children with malocclusion. J orthod, v. 34, n. 3, p. 185-193, 2007.

OLIVEIRA, B.H.; MILBOURNE, P. Fluorose dentária em incisivos superiores permanentes em crianças de escola pública do Rio de Janeiro, RJ. Rev saúde pública, v. 35, n. 3, p. 276-282, 2001.

OLIVEIRA, C.M.; SHEIHAM, A. Orthodontic treatment and its impact on oral healthrelated quality of life in Brazilian adolescents. Jorthod, v. 31, n. 1, p. 20-27, 2004.

OLIVEIRA, C.M.; SHEIHAM, A. The relationship between normative orthodontic treatment need and oral health-related quality of life. Community dent oral epidemiol, v. 31, n. 6, p. 426-436, 2003.

PAHEL, B.T.; ROZIER, R.G.; SLADE, G.D. Parental perceptions of children's oral health: the Early Childhood Oral Health Impact Scale (ECOHIS). Health qual life outcomes, v. 30, p. 6, 2007.

PAL, D.K. Quality of life assessment in children: a review of conceptual and methodological issues in multidimensional health status measures. J epidemiol community health, v. 50, n. 4, p. 391-396, 1996.

PETERSEN, P.E. The World Oral Health Report 2003: continuous improvement of oral health in the 21st century - the approach of the WHO Global Oral Health Programme. Community dent oral epidemiol, v. 31, p. 3-23, 2003.

PINTO, V.G. Saúde bucal coletiva. São Paulo: Ed. Santos, 2000.

REISINE, S.T. Theoretical considerations in formulating sociodental indicators. Soc sci med [A], v. 15, n. 6, p. 745-750, 1981.

RUPERTO, N. et al. Cross-cultural adaptation and psychometric evaluation of the Childhood Health Assessment Questionnaire (CHAQ) and the Child Health Questionnaire (CHQ) in 32 countries. Review of the general methodology. Clin exp rheumatol, v. 19, n. 4, p. 1-9, 2001.

SEIDL, E.M.; ZANNON, C.M.L.C. Qualidade de vida e saúde: aspectos conceituais e metodológicos. Cad saúde pública, v. 20, n. 2, p. 580-588, 2004.

SEVENHUYSEN, G.P.; TRUMBLE-WADDELL, J. A new perspective on quality of life. J clin epidemiol, v. 50, n. 3, p. 231-232, 1997.

SHEIHAM, A.; MAIZELS, J.E.; CUSHING, A.M. The concept of need in dental care. Int dent J, v. 32, n. 2, p. 265-270, 1982. 
SLADE, G.D. Derivation and validation of a shortform Oral Health Impact Profile. Community dent oral epidemiol, v. 25, p. 284-290, 1997a.

SLADE, G.D. Oral impacts on daily performances: measuring oral health and quality of life. North Carolina: University of North Carolina, $1997 \mathrm{~b}$.

SLADE, G.D.; SPENCER, A.J. Development and evaluation of the Oral Health Impact Profile. Community dent health, v. 11, n. 1, p. 3-11, 1994.

STEIN, R.E.; JESSOP, D.J. The impact on family scale revisited: further psychometric data. J dev behav pediatr, v. 24, n. 1, p. 9-16, 2003.

TESCH, F.C.; DE OLIVEIRA, B.H.; LEÃO, A. Equivalência semântica da versão em português do instrumento Early Childhood Oral Health Impact Scale. Cad saúde pública, v. 24, n. 8, p. 1897-1909, 2008.

TESCH, F.C.; DE OLIVEIRA, B.H.; LEĀO, A. Mensuração do impacto dos problemas bucais sobre a qualidade de vida de crianças: aspectos conceituais e metodológicos. Cad. saúde pública, v. 23, n. 11, p. 2555-2564, 2007.

TRAEBERT, J. et al. Prevalência, necessidade de tratamento e fatores predisponentes do traumatismo na dentição permanente de escolares de 11 a 13 anos de idade. Cad saúde pública, v. 20, n. 2, p. 403-410, 2004.

TUBERT-JEANNIN, S. et al. Validation of a French version of Child-OIDP index. Eur J oral sci, v. 113, n. 5, p. 355-362, 2005.

VARGAS, C.M.; CRALL, J.J.; SCHNEIDER, D.A. Sociodemographic distribution of pediatric dental caries: NHANES III, 1988-1994. J Am dent assoc, v. 129, n. 9, p. 1229-1238, 1998.

WALLANDER, J.L.; SCHMITT, M.; KOOT, H.M. Quality of life measurement in children and adolescents: issues, instruments, and applications. J clin psychol, v. 57, n. 4, p. 571-585, 2001.

WARE JR, J.E.; SHERBOURNE, C.D. The MOS 36-item short-form health survey (SF36). I. Conceptual framework and item selection. Med care, v. 30, n. 6, p. 473-483, 1992.

WEINTRAUB, J.A. Uses of oral health related quality of life measures in public health. Community dent health, v. 15, n. 1, p. 8-12, 1998.

WHOQOL GROUP. The World Health Organization Quality of Life assessment (WHOQOL): position paper from the World Health Organization. Soc sci med, v. 41, n. 10, p. 1403-1409, 1995.

WORLD HEALTH ORGANIZATION. WHOQOL:measuring quality of life. The World Health Organization quality of life instruments. Geneva: World Health Organization, 1997. YUSUF, H. et al. Validation of an English version of the Child-OIDP index, an oral healthrelated quality of life measure for children. Health qual life outcomes, v. 4, n. 38, 2006. 
Quality of life and oral health in children and adolescents: conceptual and methodological aspects

There has been increasing interest in the oral healthrelated quality of life of children and adolescents, since pediatric oral disorders are likely to have a negative effect on children's quality of life. So the questionnaires that aim to assess the impact of oral health on child' and adolescent' quality of life has been developed or adapted to this specific group. The aim of this study was to describe the relationship between the quality of life and oral health in children and adolescents, focalizing conceptual and methodological aspects. The instruments developed to assess oral health-related quality of life in children and adolescents are: the Child Perceptions Questionnaire (CPQ), for children aged 8-10 $\left(\mathrm{CPQ}_{8-10}\right)$ and 11-14 $\left(\mathrm{CPQ}_{11-14}\right)$, the Child-Oral Impacts on Daily Performances, for children aged 11-12, the Child Oral Health Impact Profile, for children aged 8-14, and the Early Childhood Oral Health Impact Scale for children aged 2-5. Although progress was observed in this area, there are still many issues to be discussed and answered, as the need for specific questionnaires for different ages, the participation of respondents as secondary and evaluation of the impact of child' and adolescent' oral health in the family.

Key words: adolescent; child; quality of life; oral health. 